\title{
Effects of an Acetic Acid and Acetone Mixture on the Characteristics and Scaffold-Cell Interaction of Electrospun Polycaprolactone Membranes
}

\author{
Minh Hieu Ho ${ }^{1}$, Thien Bui-Thuan Do ${ }^{1}$, Nhi Ngoc-Thao Dang ${ }^{1}$, An Nguyen-My Le ${ }^{1}$, \\ Hanh Thi-Kieu Ta ${ }^{2,3}$, Toi Van Vo ${ }^{1}$ and Hiep Thi Nguyen ${ }^{1, *}$ \\ 1 Tissue Engineering and Regenerative Medicine Laboratory, School of Biomedical Engineering, International \\ University, Vietnam National University, Ho Chi Minh City 700000, Vietnam; \\ hohieuminh96@gmail.com (M.H.H.); thuanthien1095@gmail.com (T.B.-T.D.); \\ dntnhi@hcmiu.edu.vn (N.N.-T.D.); Inman@hcmiu.edu.vn (A.N.-M.L.); vvtoi@hcmiu.edu.vn (T.V.V.) \\ 2 Faculty of Materials Science and Technology, University of Science, Vietnam National University, \\ Ho Chi Minh City 700000, Vietnam; ttkhanh@hcmus.edu.vn \\ 3 Center for Innovative Materials and Architectures, Vietnam National University, \\ Ho Chi Minh City 700000, Vietnam \\ * Correspondence: nthiep@hcmiu.edu.vn
}

Received: 13 September 2019; Accepted: 5 October 2019; Published: 16 October 2019

check for updates

\begin{abstract}
Green electrospinning has attracted great interest since non-toxic solvents were shown to be applicable in the fabrication of fibrous materials while ensuring health safety and environmental protection. Less harmful reagents such as acetone (AC) and acetic acid (AA) have been employed in this field in recent years. However, research in this area is still rare, yielding only preliminary results. In this study, two different types of solvents (pure AC and an AA/AC mixture) were used to fabricate electrospun polycaprolactone (PCL) membranes. Sample morphology, wettability, tensile strength, and chemical composition were compared between two types of membranes. Cell-scaffold interaction was also examined by cell adhesion and proliferation assays. The results demonstrate that the two types of solvents had significant effects on membrane morphology, physical strength, and cell adherence behaviors, which should be considered for different application purposes.
\end{abstract}

Keywords: electrospinning; polycaprolactone; acetone; acetic acid; cell adhesion; cell proliferation

\section{Introduction}

Electrospun polycaprolactone (PCL) membranes have been broadly applied in biomedical engineering due to their excellent biocompatibility, high mechanical strength, and slow degradation rate [1,2]. Common solvents used to dissolve PCL pellets for electrospinning include dimethylformamide, tetrahydrofuran, chloroform. In fact, these types of solvent are extensively employed because they are considered good solvents for obtaining a fine membrane morphology (bead-free and smooth fibers) and avoiding clogging at the needle tip, which is ideal for automatic operation and massive production [3]. However, these solvents are extremely devastating to human health and harmful to the living environment $[4,5]$. Alternatively, relatively safe chemicals such as acetone (AC) and acetic acid (AA) were discovered to be applicable for electrospinning of PCL [6]. Unlike AC which can be used as an independent solvent for PCL, studies suggest using AA in combination with other solvents at appropriate ratios rather than in pure form to avoid non-fiber formation [7] or eliminate needle-tip clogging [8]. Furthermore, the use of AA in solvents to dissolve PCL can allow the co-electrospinning of PCL and natural polymers such as chitosan [9], gelatin [10], and collagen [11] using only one single homogenous electrospinning solution rather than two solutions, because these polymers are also 
easily dissolved in AA. Compared to the toxic solvents mentioned above, AC and AA are much less detrimental to human health, showing potential as alternative solvents in electrospinning. However, research on PCL membranes using solvents containing AC and AA and systematic comparisons between them are still sparse, and results are strongly dependable on each electrospinning apparatus design. In particular, no research has been done on cell-material interaction, which has impeded our understanding of their potential applications in various areas including the biomedical, clinical, and surgical fields.

In this study, electrospun PCL membranes were fabricated using two different types of eco-friendly solvents containing AC and AA, whose effects on cell adhesion and proliferation were preliminarily examined in relation to membrane morphology and its wettability, chemical, and physical characteristics.

\section{Materials and Methods}

\subsection{Materials}

Acetone (99\%), acetic acid (99.5\%), and ethanol (99.7\%) were purchased from Xilong Chemical Co. Ltd., Shantou, China. Poly ( $\varepsilon$-caprolactone) (PCL, Mn 80.000), glutaraldehyde (25\%), and resazurin (80\%) were purchased from Sigma-Aldrich Co. Ltd., Dorset Gillingham, UK.

\subsection{Methods}

\subsubsection{Fabrication of Electrospun Membranes}

PCL pellets were dissolved in pure AC (solvent I, PCL-AC) and in a mixture of AA/AC at a ratio of 7:3 v/v (solvent II, PCL-7AA/3AC). The electrospinning parameters were pre-investigated to obtain bead-free fibers for both types of solvents. Briefly, $10 \mathrm{~mL}$ of $15 \% \mathrm{w} / \mathrm{v}$ PCL solution was horizontally electrospun at $15 \mathrm{kV}$ voltage, $1 \mathrm{~mL} / \mathrm{h}$ flow rate (55-2226, Harvard Apparatus 22, USA) onto a stationary drum collector at $10 \mathrm{~cm}$ distance.

\subsubsection{Sample Characterization}

Fiber diameter and pore size of the PCL membranes were determined by Scanning Electron Microscope (SEM, JSM-IT100, JEOL Ltd., Japan) at 3 separate locations and 10 measurements per location. To be specific, the values of pore size were represented by the diameter of the inscribed circle of each triangle formed by three intersecting fibers. For wettability analysis, a high-resolution camera was used to capture the image of a water drop on the membrane surface, and the contact angle was determined using Image J. Stress-strain curves were obtained (TA.XTplus, Stable Micro Systems Co., $\mathrm{UK}$ ) to examine the physical strength of the membranes. Chemical compositions were examined by Fourier-Transform Infrared Spectroscopy (FT-IR, Spectrum GX, PerkinElmer Inc., USA).

\subsubsection{Cell Attachment and Proliferation Assays}

The membranes $\left(1.5 \times 1.5 \mathrm{~cm}^{2}\right)$ were sterilized in ethanol $70 \%$ for $4 \mathrm{~h}$ and exposed under UV light for $1 \mathrm{~h}$ per each surface.

For the cell adhesion assay, $1 \times 10^{5}$ of murine skin fibroblasts L929s in $1 \mathrm{~mL}$ media were seeded onto the top surface of the membranes. After $4 \mathrm{~h}$, the membranes were washed with PBS, fixed in glutaraldehyde $4 \%$ for $20 \mathrm{~min}$ and an increasing series of ethanol, 50\%, 60\%, 70\%, 80\%, 90\%, 95\% (3 min each) and 100\% (3 times, 10 min each) for SEM analysis.

For the cell proliferation assay, a special cell culture tool was designed and 3D-printed from poly(lactic-co-glycolic acid) to avoid the floating of the membranes in cell culture medium (Figure 1). A total of $5 \times 10^{4}$ of L929 cells in $1 \mathrm{~mL}$ of medium were seeded onto the membrane surface through the upper open end of the tool. Culture medium was also placed in the culture well and changed every 2 days. At day 1,3,5, and 7, the membranes were transferred to a new 24 -well plate, and $1 \mathrm{~mL}$ 
of medium containing $100 \mu \mathrm{L}$ of resazurin solution $(0.2 \mathrm{mg} / \mathrm{mL})$ was added to measure the cellular metabolic activity in relation to the cell population on the membrane. After $4 \mathrm{~h}$ incubation, fluorescent intensity in each well was recorded at Ex $530 \mathrm{~nm} / \mathrm{Em} 590 \mathrm{~nm}$ (Varioskan LUX, Thermo Fisher, USA). The experiment was done in triplicate for statistical analysis.

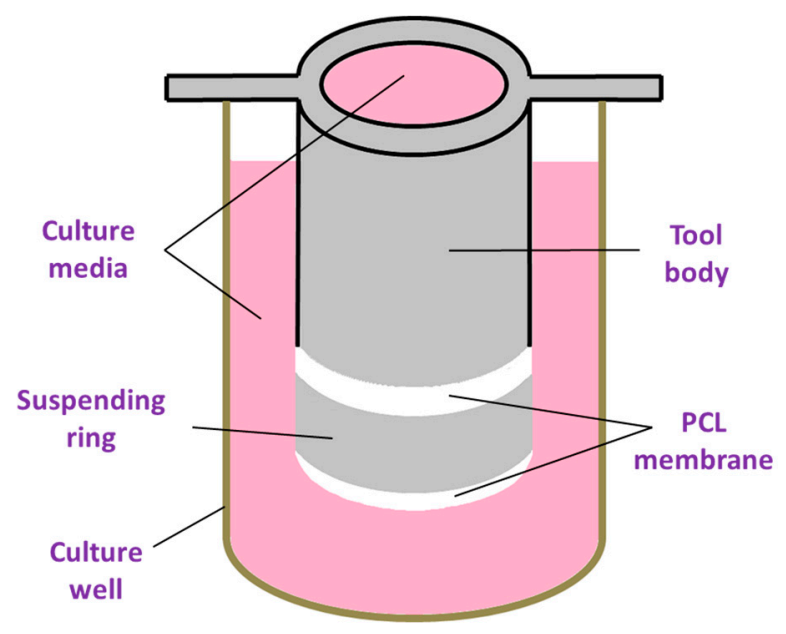

Figure 1. Schematic of the 3D-printed cell culture tool.

\section{Results and Discussion}

\subsection{Surface Morphology}

Figure 2 shows that in general, there was a significant difference in fiber diameter and pore size between the two membranes. On average (Figure 2B), the PCL-AC membrane possessed a fiber diameter that was approximately 4 times larger and pore size 7 times bigger in comparison to the PCL-7AA/3AC membrane. In particular, histograms illustrated an irregular trend in the distribution of fiber diameter and pore size in the PCL-AC membrane. The most frequent fiber diameter values were in three distinct ranges: 500-1000, 1500-2000, and 3000-3500 nm, while the dominant pore sizes ranged broadly from 6 to $14 \mu \mathrm{m}$. For the PCL-7AA/3AC membrane, more than $75 \%$ of fibers were less than $500 \mu \mathrm{m}$ in diameter, and the dominant pore sizes fell between 1.2 and $1.8 \mu \mathrm{m}$. Thus, it was clearly noticed that the addition of AA dramatically reduced the fiber diameter from the microscale to the nanoscale, which was also found in other reports [7,12]. This could be attributed to the reported association between viscosity of the polymer solution and average fiber diameter [13]. At the same PCL concentration, the addition of AA in the solvent decreased the viscosity of the polymer solution compared to when using acetone alone [8]. This led to the increase in the solution's surface tension pointing inward and narrowing the fiber width [14]. Furthermore, another difference was the increase in conductivity of the PCL solution when using the AA/AC solvent. PCL chains carrying a negative charge from the carboxylic group of AA accelerated the stretching, elongation, and thinning of the PCL fibers [3]. The combination of the surface tension force of the solution and the repulsive electrostatic force among PCL chains resulted in a significant decrease in the fiber diameter of the PCL-7AA/3AC membrane [3]. On the other hand, the erratic size of PCL-AC fibers could be attributed to the fact that solution clogging occurred at the needle tip, which was not seen for the PCL-7AA/3AC membranes. This was due to a higher evaporation rate of $\mathrm{AC}$ compared to AA, relative to the solution feeding rate [15]. The excessive amount of polymer accumulating at the needle tip resulted in the formation of an unstable Taylor cone and thus produced larger fibers in the PCL-AC membrane [8]. 
(A)

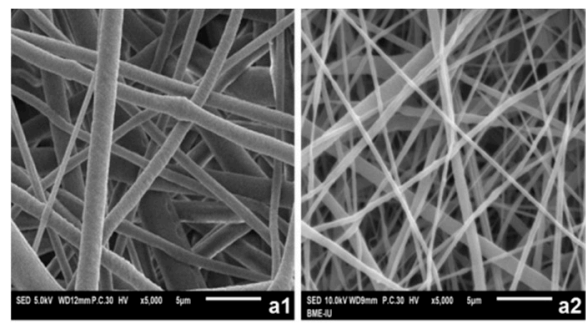

\begin{tabular}{|c|c|c|}
\hline Membrane & $\begin{array}{c}\text { Fiber diameter } \\
(\mu \mathrm{m})\end{array}$ & $\begin{array}{c}\text { Pore size } \\
(\mu \mathrm{m})\end{array}$ \\
\hline $\mathrm{PCL}-\mathrm{AC}$ & $1.78 \pm 1.07$ & $11.31 \pm 3.54$ \\
\hline $\mathrm{PCL}-7 \mathrm{AA} / 3 \mathrm{ACC}$ & $0.45 \pm 0.29$ & $1.63 \pm 0.41$ \\
\hline
\end{tabular}

(B)
(C)
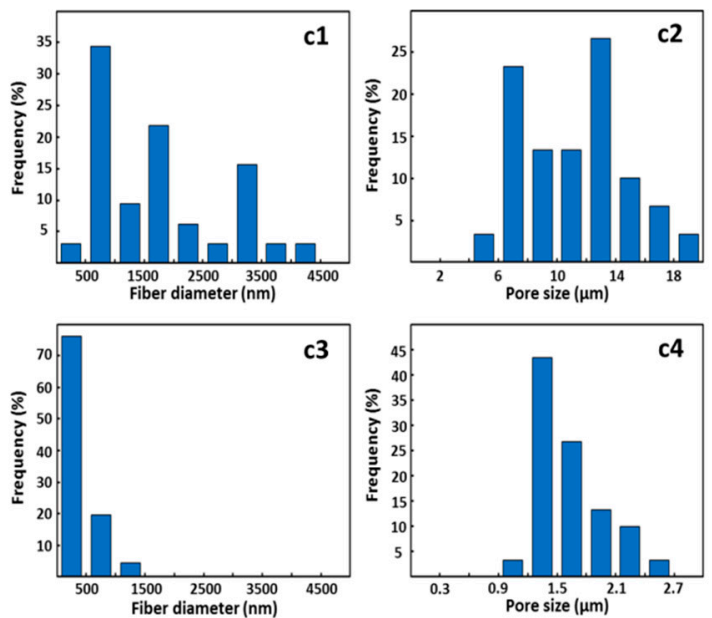

Figure 2. (A) Scanning Electron Microscope (SEM) images of electrospun polycaprolactone (PCL) in acetone (AC), (PCL-AC), (a1) and PCL dissolved in acetic acid (AA)/AC (7:3, PCL-7AA/3AC) (a2) membranes, $(B)$ Average fiber diameter and pore size, and $(C)$ Histogram of fiber diameter and pore size distribution of PCL-AC (c1,c2) and PCL-7AA/3AC (c3,c4) membranes.

\subsection{Wettability, Chemical, and Physical Properties}

In Figure 3, wettability test and FT-IR results displayed no significant difference between the two membranes. Both PCL-AC and PCL-7AA/3AC surfaces were hydrophobic, with average contact angle values of $118^{\circ}$ and $116^{\circ}$, respectively. The chemical composition of the two membranes was the same, with similar dominant peaks characteristic of PCL at 2940, 2864, 1722, and $1240 \mathrm{~nm}$ [16]. No distinctive peaks of AC or AA were found for either of the two membranes, demonstrating that the solvents were completely removed. However, a noticeable difference was encountered in terms of physical strength. The PCL-AC membrane could endure up to $12.4 \mathrm{MPa}$ before being torn apart completely. The deformation ratio at break of the PCL-AC membrane was about $167 \%$ of its original length. Meanwhile, the PCL-7AA/3AC membrane possessed far weaker strength, could endure only $7.8 \mathrm{MPa}$, and its maximum strain was $60 \%$. Reports have shown that there is a relationship between fiber diameter and tensile strength. In contrast to the PCL-7AA/3AC membrane with low tensile strength, the PCL-AC membrane consisted of thicker fibers with fibers point bonding and some fusion of adjacent fibers [17], which increased the fiber interconnectivity and therefore resulted in higher tensile strength [18]. In another study, A. R. Hernández found that PCL was hydrolyzed and degraded into oligomeric fragments in both alkaline and acidic solutions [19]. Therefore, the presence of the carboxylic group of AA in the solvent could aid in cutting down the long PCL chains into shorter ones [20] with lower molecular weights, and this helps explain the significant decrease in tensile strength of the PCL-AA membranes [21]. 


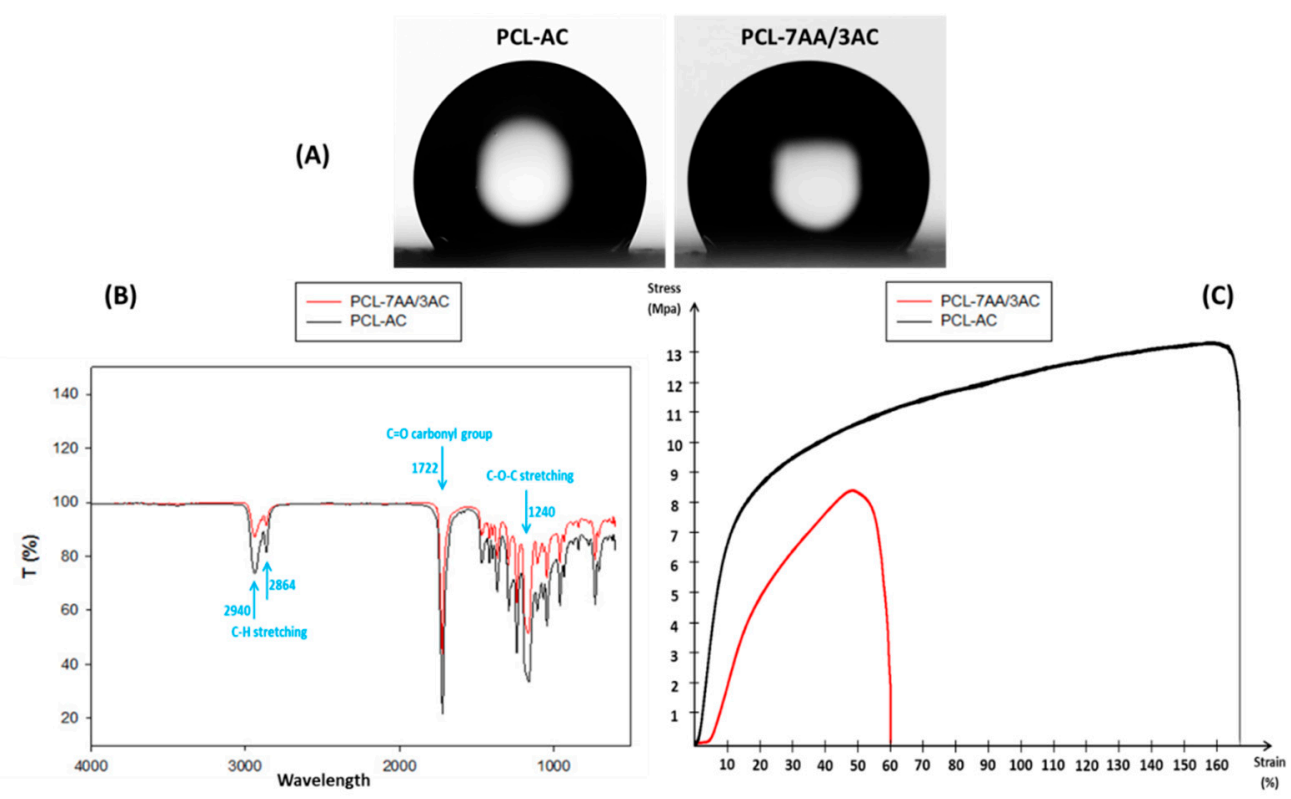

Figure 3. (A) Water contact angle, (B) FT-IR spectra, and (C) Tensile curves of PCL-AC and PCL-7AA/3AC membranes.

\subsection{In Vitro Studies}

Figure 4 illustrates the cell-material interaction for the two types of membrane. SEM images show that a very small number of cells were found on the surface of the PCL-AC membrane. Regarding the morphology, the cells were not able to spread but shrank to nearly round shapes. In contrast, the PCL-7AA/3AC membrane displayed a higher significant number of L929 cells adhering on the surface, which were fully stretched and, hence, larger in size. As shown by the proliferation assay (Figure 4B), the proliferation rate calculated from the fluorescent signal every 2 days was approximately equal for the two types of membrane. This indicates that the influence of the two types of membrane on biocompatibility and cell proliferation rate was not significantly different. However, the number of cells found on the PCL-7AA/3AC membrane was always higher than the number of cells found on the PCL-AC membrane at all time points. This could be due to the clear difference in the number of cells initially adherent to the surface, which was supported by SEM images. These results indicate that the PCL-7AA/3AC membrane was more supportive of cell attachment than the PCL-AC membrane. This agrees with other studies that showed that the smaller pore size of the PCL-7AA/3AC membranes increases the surface area, increasing the surface for initial cell attachment [22]. Although the larger fiber diameters in the PCL-AC membrane also increased the surface area for cell adhesion, this effect might have been restricted by the hydrophobicity of the fibers [23] and outweighed by the large mean pore size [24]. Moreover, compared to the size of L929 cell pellets shortly post-seeding $(8-10 \mu \mathrm{m})$ [25], the PCL-AC pores were larger, while the PCL-7AA/3AC membrane acted as a barrier, retaining the cells on the surface. This indicates that the seeded cell pellets might infiltrate directly through the large pores of the PCL-AC sample [26] and helps explain the equal proliferative rates of the two membranes, which needs to be examined further in subsequent studies. 
(A)

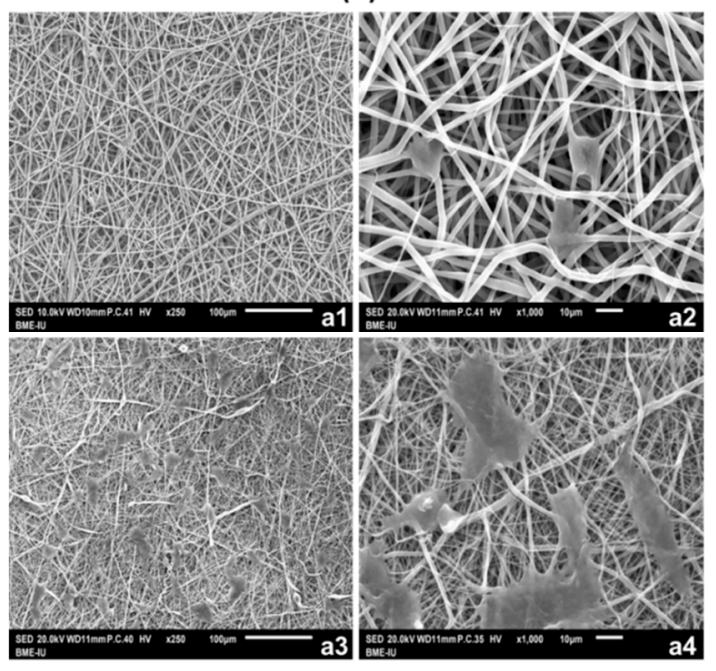

(B)

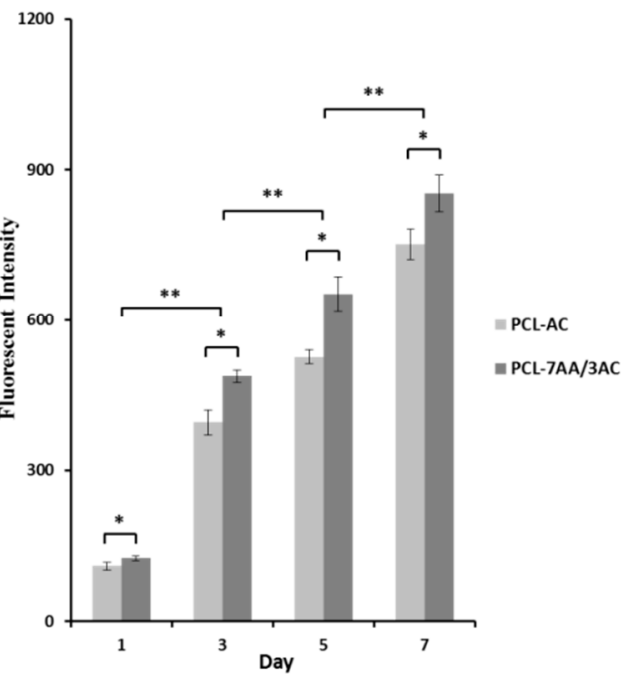

Figure 4. (A) SEM images of L929 cell attachment on PCL-AC (a1, a2) and PCL-7AA/3AC (a3, a4) membranes after $4 \mathrm{~h}$ incubation and (B) Proliferation of L929 cells on PCL-AC and PCL-7AA/3AC membranes for $1,3,5,7$ days.

First and foremost, the use of relatively safe solvents like AC and AA is beneficial and sustainable, in line with the search for green solutions in all fields, including electrospinning research, to eliminate the harmful effects of toxic organic solvents on human health and the living environment. The presented results indicate that the applications of the two fabricated membranes can be diverse. The clinical study of Khan et al. reported that although sample extracts showed no sign of toxicity in vivo, chitosan films prepared from AA caused skin irritation in patients due to the acetate anions remaining on the films [27]. As demonstrated in Figure 3B, the solvent was completely removed from the PCL-7AA/3AC membrane during the electrospinning process, which implies a possible use of the 7AA/3AC mixed solvent in co-electrospinning of PCL and other natural polymers like chitosan, gelatin, and collagen for clinical applications such as wound dressings, without causing allergy. In addition, the small pore size of the PCL-7AA/3AC membrane suggests its potential clinical and surgical use as a barrier membrane, for example in guided bone regeneration for atrophic jaw reconstruction [28]. For this purpose, PCL-7AA/AC was effective not only in retaining the cells on the surface but also in supporting cell adhesion and proliferation, an important and necessary factor determining the success of a barrier membrane in guided bone regeneration [29]. Meanwhile, the high tensile strength and hydrophobic properties of the PCL-AC membrane suggest its applications in the textile industry, for example, for waterproof clothing. In biomedical engineering, the PCL-AC membrane with big pore sizes suitable for cell infiltration can be used as a scaffold for the fabrication of artificial tissues [30-32] or as a template for wound healing applications $[2,33]$.

\section{Conclusions}

In this study, different types of solvents were shown to have significant effects on the morphology of PCL membranes and thus affect cell-membrane interaction. Utilizing pure AC as a solvent yielded microfibers and large pore size with high stress-strain values. Meanwhile, although the mixture of 7AA/3AC used as a solvent reduced the fiber size to the nanoscale, supporting initial cell adhesion, the fibers had significantly lower tensile strength as a tradeoff.

Author Contributions: M.H.H., conceptualization, methodology, investigation, validation, writing-original draft preparation, writing-review and editing; T.B.-T.D., investigation, writing-review and editing; N.N.-T.D., visualization; A.N.-M.L., resources; H.T.-K.T., formal analysis; T.V.V., project administration; H.T.N., supervision, funding acquisition. 
Funding: This research was funded by National Foundation for Science and Technology Development (NAFOSTED, Vietnam) under grant number 108.06-2018.18.

Conflicts of Interest: The authors declare no conflict of interest.

\section{References}

1. Minh, H.H.; Hiep, N.T.; Hai, N.D.; Van Toi, V. Fabrication of Polycaprolactone/Polyurethane Loading Conjugated Linoleic Acid and Its Antiplatelet Adhesion. Int. J. Biomater. 2017, 2017, 5690625. [CrossRef]

2. Thanh, N.T.; Hieu, M.H.; Phuong, N.T.M.; Thuan, T.D.B.; Thu, H.N.T.; Thai, V.P.; Minh, T.D.; Dai, H.N.; Vo, V.T.; Thi, H.N.; et al. Optimization and characterization of electrospun polycaprolactone coated with gelatin-silver nanoparticles for wound healing application. Mater. Sci. Eng. 2018, 91, 318-329. [CrossRef] [PubMed]

3. Haider, A.; Haider, S.; Kang, I.-K. A comprehensive review summarizing the effect of electrospinning parameters and potential applications of nanofibers in biomedical and biotechnology. Arab. J. Chem. 2018, 11, 1165-1188. [CrossRef]

4. Meek, M.E.; Long, G. N,N-dimethylformamide: Hazard characterization and exposure-response analysis. J. Environ. Sci. Health Part C 2001, 19, 161-187.

5. Fowles, J.; Boatman, R.; Bootman, J.; Lewis, C.; Morgott, D.; Rushton, E.; Van Rooij, J.; Banton, M. A review of the toxicological and environmental hazards and risks of tetrahydrofuran. Crit. Rev. Toxicol. 2013, 43, 811-828. [CrossRef] [PubMed]

6. Ekram, B.; Abdel-Hady, B.M.; El-kady, A.M.; Amr, S.M.; Waley, A.I.; Guirguis, O.W. Optimum parameters for the production of nano-scale electrospun polycaprolactone to be used as a biomedical material. Adv. Nat. Sci. Nanosci. Nanotechnol. 2017, 8, 045018. [CrossRef]

7. Van der Schueren, L.; De Schoenmaker, B.; Kalaoglu, Ö.I.; De Clerck, K. An alternative solvent system for the steady state electrospinning of polycaprolactone. Eur. Polym. J. 2011, 47, 1256-1263. [CrossRef]

8. Augustine, R.; Kalarikkal, N.; Thomas, S. Clogging-Free Electrospinning of Polycaprolactone Using Acetic Acid/Acetone Mixture. Polym. Plast. Technol. Eng. 2016, 55, 518-529. [CrossRef]

9. Cooper, A.; Bhattarai, N.; Zhang, M. Fabrication and cellular compatibility of aligned chitosan-PCL fibers for nerve tissue regeneration. Carbohydr. Polym. 2011, 85, 149-156. [CrossRef]

10. Gautam, S.; Dinda, A.K.; Mishra, N.C. Fabrication and characterization of PCL/gelatin composite nanofibrous scaffold for tissue engineering applications by electrospinning method. Mater. Sci. Eng. C Mater. Biol. Appl. 2013, 33, 1228-1235. [CrossRef]

11. Hackett, J.M.; Dang, T.T.; Tsai, E.C.; Cao, X. Electrospun Biocomposite Polycaprolactone/Collagen Tubes as Scaffolds for Neural Stem Cell Differentiation. Materials 2010, 3, 3714-3728. [CrossRef]

12. Enis, I.Y.; Vojtech, J.; Sadikoglu, T.G. Alternative solvent systems for polycaprolactone nanowebs via electrospinning. J. Ind. Text. 2017, 47, 57-70. [CrossRef]

13. Cramariuc, B.; Cramariuc, R.; Scarlet, R.; Manea, L.R.; Lupu, I.G.; Cramariuc, O. Fiber diameter in electrospinning process. J. Electrost. 2013, 71, 189-198. [CrossRef]

14. Pan, Y.; Zeng, L. Simulation and Validation of Droplet Generation Process for Revealing Three Design Constraints in Electrohydrodynamic Jet Printing. Micromachines (Basel) 2019, 10, 94. [CrossRef]

15. Ghorani, B.; Russell, S.J.; Goswami, P. Controlled Morphology and Mechanical Characterisation of Electrospun Cellulose Acetate Fibre Webs. Int. J. Polym. Sci. 2013, 2013, 12. [CrossRef]

16. Abderrahim, B.; Abderrahman, E.; Mohamed, A.; Fatima, T.; Abdesselam, T.; Krim, O. Kinetic Thermal Degradation of Cellulose, Polybutylene Succinate and a Green Composite: Comparative Study. World J. Environ. Eng. 2015, 3, 95-110.

17. Asvar, Z.; Mirzaei, E.; Azarpira, N.; Geramizadeh, B.; Fadaie, M. Evaluation of electrospinning parameters on the tensile strength and suture retention strength of polycaprolactone nanofibrous scaffolds through surface response methodology. J. Mech. Behav. Biomed. Mater. 2017, 75, 369-378. [CrossRef]

18. Kancheva, M.; Toncheva, A.; Manolova, N.; Rashkov, I. Enhancing the mechanical properties of electrospun polyester mats by heat treatment. Express Polym. Lett. 2015, 9, 49-65. [CrossRef]

19. Hernández, A.R.; Contreras, O.C.; Acevedo, J.C.; Moreno, L.G.N. Poly( $(\varepsilon$-caprolactone) Degradation Under Acidic and Alkaline Conditions. Am. J. Polym. Sci. 2013, 3, 70-75. 
20. Bosworth, L.A.; Downes, S. Physicochemical characterisation of degrading polycaprolactone scaffolds. Polym. Degrad. Stab. 2010, 95, 2269-2276. [CrossRef]

21. Shrivastava, A. 2-Polymerization. In Introduction to Plastics Engineering; Shrivastava, A., Ed.; William Andrew Publishing: Norwich, NY, USA, 2018; pp. 17-48.

22. Murphy, C.M.; Haugh, M.G.; O'Brien, F.J. The effect of mean pore size on cell attachment, proliferation and migration in collagen-glycosaminoglycan scaffolds for bone tissue engineering. Biomaterials 2010, 31, 461-466. [CrossRef]

23. Shafei, S.; Foroughi, J.; Chen, Z.; Wong, C.S.; Naebe, M. Short Oxygen Plasma Treatment Leading to Long-Term Hydrophilicity of Conductive PCL-PPy Nanofiber Scaffolds. Polymers 2017, 9, 614. [CrossRef]

24. Elsayed, Y.; Lekakou, C. 2-Designing and modeling pore size distribution in tissue scaffolds. In Characterisation and Design of Tissue Scaffolds; Tomlins, P., Ed.; Woodhead Publishing: Cambridge, UK, 2016; pp. 23-43.

25. Vogler, M.; Vogel, S.; Krull, S.; Farhat, K.; Leisering, P.; Lutz, S.; Wuertz, C.M.; Katschinski, D.M.; Zieseniss, A. Hypoxia modulates fibroblastic architecture, adhesion and migration: A role for HIF-1 $\alpha$ in cofilin regulation and cytoplasmic actin distribution. PLoS ONE 2013, 8, e69128. [CrossRef] [PubMed]

26. Ko, Y.-G.; Park, J.H.; Lee, J.B.; Oh, H.H.; Park, W.H.; Cho, D.; Kwon, O.H. Growth behavior of endothelial cells according to electrospun poly(D,L-lactic-co-glycolic acid) fiber diameter as a tissue engineering scaffold. Tissue Eng. Regen. Med. 2016, 13, 343-351. [CrossRef] [PubMed]

27. Khan, T.A.; Peh, K.K.; Ch'ng, H.S. Mechanical, bioadhesive strength and biological evaluations of chitosan films for wound dressing. J. Pharm. Pharm. Sci. 2000, 3, 303-311.

28. Cicciu, M. Real Opportunity for the Present and a Forward Step for the Future of Bone Tissue Engineering. J. Craniofac. Surg. 2017, 28, 592-593. [CrossRef] [PubMed]

29. Soldatos, N.K.; Stylianou, P.; Koidou, V.P.; Angelov, N.; Yukna, R.; Romanos, G.E. Limitations and options using resorbable versus nonresorbable membranes for successful guided bone regeneration. Quintessence Int. (Berlin Ger. 1985) 2016, 48, 131-147.

30. Semnani, D.; Naghashzargar, E.; Hadjianfar, M.; Dehghan Manshadi, F.; Mohammadi, S.; Karbasi, S.; Effaty, F. Evaluation of PCL/chitosan electrospun nanofibers for liver tissue engineering. Int. J. Polym. Mater. Polym. Biomat. 2017, 66, 149-157. [CrossRef]

31. Mohseni, M.; Castro, N.J.; Dang, H.P.; Nguyen, T.D.; Ho, H.M.; Tran, M.P.N.; Nguyen, T.H.; Tran, P.A. 13-Adipose tissue regeneration: Scaffold-Biomaterial strategies and translational perspectives. In Biomaterials in Translational Medicine; Yang, L., Bhaduri, S.B., Webster, T.J., Eds.; Academic Press: Cambridge, MA, USA, 2019; pp. 291-330.

32. Sundaramurthi, D.; Krishnan, U.M.; Sethuraman, S. Electrospun Nanofibers as Scaffolds for Skin Tissue Engineering. Polym. Rev. 2014, 54, 348-376. [CrossRef]

33. Han, F.; Zhang, P.; Sun, Y.; Lin, C.; Zhao, P.; Chen, J. Hydroxyapatite-doped polycaprolactone nanofiber membrane improves tendon-bone interface healing for anterior cruciate ligament reconstruction. Int. J. Nanomed. 2015, 10, 7333-7343.

(C) 2019 by the authors. Licensee MDPI, Basel, Switzerland. This article is an open access article distributed under the terms and conditions of the Creative Commons Attribution (CC BY) license (http://creativecommons.org/licenses/by/4.0/). 